\title{
Coupled Optical Interface Modes in a Thue-Morse Dielectric Superlattice
}

\author{
Sheng-Feng Cheng, Guo-Jun Jin, Ru-Wen Peng and An Hu
}

\author{
National Laboratory of Solid State Microstructures and Department of Physics, Nanjing University, \\ Nanjing 210093, People's Republic of China
}

(Received November 20, 2000)

\begin{abstract}
We study the coupled optical interface modes in a Thue-Morse dielectric superlattice. Based on the electrostatic continuum approximation, which is correct in the long wavelength limit, the transfer matrix method is used to derive the analytic expressions for frequency spectra and electrostatic potential distributions. The numerical calculations show that the frequency spectra are singular continuous, and the potential profiles are mostly extended and lattice-like. The common features and pronounced differences with coupled optical interface modes in periodic and Fibonacci dielectric superlattices as well as with electrons and acoustic phonons in Thue-Morse structures are exposed. Furthermore, the possibility of experimental observation is discussed.
\end{abstract}

KEYWORDS: Thue-Morse sequence, optical phonons, dielectric superlattice

\section{§1. Introduction}

Since the discovery of a quasicrystalline phase in $\mathrm{Al}-$ Mn alloys, great experimental and theoretical efforts have been devoted to the physical properties of onedimensional quasiperiodic structures. ${ }^{1-5)}$ These structures, known as the Fibonacci lattice, have Cantor set energy spectra and critical eigenstates. ${ }^{3)}$ Afterwards, the generalized Fibonacci ${ }^{6)}$ and other deterministic aperio$\mathrm{dic}^{7)}$ lattices have been investigated. The Thue-Morse (TM) lattice, according to its construction rule, is a deterministic aperiodic but not quasiperiodic structure, and compared with the Fibonacci lattice, it has a singular continuous Fourier transform ${ }^{8)}$ and a Cantorlike set energy spectrum. ${ }^{9)}$

On the other hand, the elementary excitations in periodic alkali halide or polar semiconductor superlattices, including acoustic phonons and optical phonons, are attractive. ${ }^{10-13)}$ Usually, the optical modes can be divided into two types: one is bulk-like excitations; the other is interface modes. Interestingly the interface modes will be coupled to give the collective excitation of the whole superlattice when the layer thicknesses in the system is relatively small. ${ }^{10,11)}$ The discovery of quasicrystals has stimulated a lot of researches in the properties of excitations in quasiperiodic superlattices. ${ }^{14-16)}$ One example is that the coupled optical interface modes in a Fibonacci superlattice had been studied. ${ }^{17)}$ One may consider that the aperiodicity of TM structures has substantial impact on the properties of elementary excitations..$^{8,9,18-23)}$ Therefore, it is worthwhile to explore the features of coupled optical interface modes in TM dielectric superlattices.

In this paper, we first introduce the dielectric continuum model for a TM superlattice and obtain the basic formulas for transfer matrices, dispersion relation and limiting frequencies. Then the numerical results of the frequency spectra, the integrated density of states (IDOS), and the electrostatic potential profiles are presented, with relevant discussions at the same time.
Finally, a brief summary is given.

\section{§2. The Dielectric Continuum Model}

We can construct a TM superlattice with two initial building blocks denoted by $L$ and $S$, and then generate it by the successive substitutions $L \rightarrow L S$ and $S \rightarrow S L$. This process, starting with $L S$, will give the following sequence: $\quad L S \rightarrow L S S L \rightarrow L S S L S L L S \rightarrow$ $L S S L S L L S S L L S L S S L \rightarrow \cdots$. To distinguish the neighboring same blocks such as $S S$ and $L L$, each block is composed of two layers with materials $A$ and $B$. So the $j$ th generation of the TM lattice has $2^{j}$ blocks and $2^{j+1}$ layers. It is assumed that the $B$ layers in $L$ and $S$ blocks have the same thickness $d$, but the $A$ layers have the thickness $d_{L}$ in $L$ blocks and $d_{S}$ in $S$ blocks. $A$ and $B$ are two kinds of dielectric materials with different dielectric functions $\varepsilon_{A}$ and $\varepsilon_{B}$, which are choose as same as those in the corresponding bulk materials and may be frequency dependent.

For a polar superlattice, in the electrostatic limit, it is proper to describe the long wavelength optical phonons by an electrostatic potential $\Phi$ satisfying the Laplace equation $\nabla^{2} \Phi(\boldsymbol{r}, t)=0 .{ }^{10)}$ To investigate the interface phonons, the $z$ axis is chosen to be perpendicular to superlattice planes, and without loss of generality, only a plane-wave $\exp (\mathrm{i} k x)$ is assumed to propagate along the $x$ direction with $k$ as the in-plane wave vector. It is reasonable to write $\Phi(\boldsymbol{r}, t)=\phi(z) \exp \{\mathrm{i}(k x-\omega t)\}$, and thus

$$
\left(\frac{\mathrm{d}^{2}}{\mathrm{~d} z^{2}}-k^{2}\right) \phi(z)=0 .
$$

Denoting $n$ as a layer index, the electrostatic continuum conditions at the interface are

$$
\phi_{n}(z)=\phi_{n+1}(z), \quad \varepsilon_{n} \frac{\mathrm{d} \phi_{n}(z)}{\mathrm{d} z}=\varepsilon_{n+1} \frac{\mathrm{d} \phi_{n+1}(z)}{\mathrm{d} z} .
$$

Following the previous discussion for a Fibonacci superlattice, ${ }^{17}$ the solution of eq. (1) for the $l$ th block can be written as $\phi_{l}(z)=p_{l} \mathrm{e}^{k z}+q_{l} \mathrm{e}^{-k z}$ in the $B$ layers, and $\phi_{l}(z)=g_{l} \mathrm{e}^{k z}+h_{l} \mathrm{e}^{-k z}$ in the $A$ layers. However, for 
mathematical convenience, a modified description must be made. Instead of considering from an $A$ layer to a subsequent $A$ layer, we write the relation between the coefficients for the $B$ layers in thelth and $(l+1)$ th blocks as

$$
\left(\begin{array}{c}
p_{l+1} \\
q_{l+1}
\end{array}\right)=T_{l+1, l}\left(\begin{array}{c}
p_{l} \\
q_{l}
\end{array}\right) .
$$

It is straightforward to obtain the $2 \times 2$ transfer matrix

$$
T_{l+1, l}=T_{l}=\left(\begin{array}{cc}
\alpha_{l} \mathrm{e}^{k d} & \beta_{l} \\
-\beta_{l} & \gamma_{l} \mathrm{e}^{-k d}
\end{array}\right),
$$

where

$$
\begin{aligned}
\alpha_{l} & =\cosh k d_{l}+\frac{1}{2}\left(\frac{\varepsilon_{A}}{\varepsilon_{B}}+\frac{\varepsilon_{B}}{\varepsilon_{A}}\right) \sinh k d_{l}, \\
\beta_{l} & =\frac{1}{2}\left(\frac{\varepsilon_{A}}{\varepsilon_{B}}-\frac{\varepsilon_{B}}{\varepsilon_{A}}\right) \sinh k d_{l}, \\
\gamma_{l} & =\cosh k d_{l}-\frac{1}{2}\left(\frac{\varepsilon_{A}}{\varepsilon_{B}}+\frac{\varepsilon_{B}}{\varepsilon_{A}}\right) \sinh k d_{l} .
\end{aligned}
$$

In the $L$ blocks $d_{l}=d_{L}, T_{l}=T_{L}$; while in the $S$ blocks $d_{l}=d_{S}, T_{l}=T_{S}$. In our arrangement, there are only two types of transfer matrices, which are all unimodular. Setting $M_{j}=\prod_{l=1}^{N} T_{l}, N=2^{j}$, we can get $M_{1}=T_{S} T_{L}$, $M_{2}=T_{L} T_{S} T_{S} T_{L}, \cdots$, and thus the global equation for a finite structure is

$$
\left(\begin{array}{l}
p_{N+1} \\
q_{N+1}
\end{array}\right)=M_{j}\left(\begin{array}{l}
p_{1} \\
q_{1}
\end{array}\right) .
$$

For substitution sequences, one is always concerned with the trace operation. As usual, by defining $\chi_{j}=$ $(1 / 2) \operatorname{Tr} M_{j}$ for $j \geq 1$, the following trace $\operatorname{map}^{7)}$

$$
\chi_{j+1}=4 \chi_{j-1}^{2}\left(\chi_{j}-1\right)+1
$$

is still hold in our case for optical interface modes. However, the recursion is a little tedious because the initial expressions for $\chi_{1}$ and $\chi_{2}$ are more complicated than for electrons ${ }^{7,20)}$ and acoustic phonons. ${ }^{23)}$ They are

$$
\chi_{1}=2 \chi_{L} \chi_{S}-\cosh k\left(d_{L}-d_{S}\right)
$$

and

$$
\chi_{2}=4 \chi_{1} \chi_{L} \chi_{S}-2 \chi_{L}^{2}-2 \chi_{S}^{2}+1
$$

with

$$
\begin{aligned}
\chi_{L(S)}= & \cosh k d_{L(S)} \cosh k d \\
& +\frac{1}{2}\left(\frac{\varepsilon_{A}}{\varepsilon_{B}}+\frac{\varepsilon_{B}}{\varepsilon_{A}}\right) \sinh k d_{L(S)} \sinh k d .
\end{aligned}
$$

Different from the Fibonacci sequence, ${ }^{17)}$ there does not exist any polynomial invariant for the TM structures, because the trace map (7) is volume-nonpreserving, ${ }^{24}$ ) i.e., its Jacobian is equal to 0 for recursion process. So we are lack of a powerful tool to judge the aperiodic behavior in TM sequences for optical phonons as well as for electrons and acoustic phonons.

Equations (7)-(10) provide us a convenient way to calculate the frequency spectra and then other properties of a TM dielectric superlattice. However, for a real TM structure, we rather here use the free-boundary condi- tions: the electrostatic potentials at the left and the right boundaries, which contact with the environment of dielectric function $\varepsilon_{C}$, are $\Phi_{L}$ and $\Phi_{R}$ with $\Phi_{L(R)}=$ $\phi_{L(R)} \exp \{\mathrm{i}(k x-\omega t)\}$. Moreover $\Phi_{L}$ and $\Phi_{R}$ are also satisfied by eq. (1). In detail, the constraint equations are written as

$$
\begin{aligned}
\left(\varepsilon_{B}-\varepsilon_{C}\right) \mathrm{e}^{-k d / 2} p_{1}-\left(\varepsilon_{B}+\varepsilon_{C}\right) \mathrm{e}^{k d / 2} q_{1} & =0, \\
\left(\varepsilon_{B}+\varepsilon_{C}\right) \mathrm{e}^{k d / 2} p_{N+1}-\left(\varepsilon_{B}-\varepsilon_{C}\right) \mathrm{e}^{-k d / 2} q_{N+1} & =0 .
\end{aligned}
$$

On the other hand, for clearness, the global equation (6) can be rewritten as

$$
\left(\begin{array}{c}
p_{N+1} \\
q_{N+1}
\end{array}\right)=M_{j}\left(\begin{array}{l}
p_{1} \\
q_{1}
\end{array}\right)=\left(\begin{array}{ll}
m_{11} & m_{12} \\
m_{21} & m_{22}
\end{array}\right)\left(\begin{array}{l}
p_{1} \\
q_{1}
\end{array}\right),
$$

where $m_{11}, m_{12}, m_{21}$, and $m_{22}$ are all complicated functions of the wave-vector, thickness and frequency. The linear equations of $p_{1}, q_{1}, p_{N+1}$ and $q_{N+1}$ in eqs. (11) and (12) have nontrivial solutions only if the coefficient determinant equals to zero. Thus the dispersion equation becomes

$$
\begin{aligned}
\left(\varepsilon_{B}+\varepsilon_{C}\right)^{2} \mathrm{e}^{k d} m_{11} & +\left(\varepsilon_{B}^{2}-\varepsilon_{C}^{2}\right) m_{12}-\left(\varepsilon_{B}^{2}-\varepsilon_{C}^{2}\right) m_{21} \\
& -\left(\varepsilon_{B}-\varepsilon_{C}\right)^{2} \mathrm{e}^{-k d} m_{22}=0
\end{aligned}
$$

From eq. (13) all relevant information regarding the optical interface modes in the present deterministic aperiodic structure can be extracted. We will see in the later numerical calculations, in a specified case, this equation is of $2 N$-th order in $\omega$, which gives $2 N$ eigenfrequencies for each value of $k$, where $N$ is related to the generation number $j$ as $N=2^{j}$.

Each of the eigenfrequencies can create a special distribution of electrostatic potential. First, we consider the amplitudes in the $B$ layers. By using eqs. (3) and (4), the potential amplitudes $p_{l+1}, q_{l+1}$ of the $l+1$ block can be recursively obtained if $p_{1}$ and $q_{1}$ are known. After $p_{l}$ and $q_{l}$ are determined, the potential distributions in the $A$ layers, characterized by $g_{l}$ and $h_{l}$, can also be obtained by using interface continuum conditions.

\section{§3. Frequency Spectra}

To get the concrete dispersion relation from eq. (13), we choose $\varepsilon_{B}$ as frequency independent, but $\varepsilon_{A}(\omega)=$ $\varepsilon_{A, \infty}\left(\omega^{2}-\omega_{A, L O}^{2}\right) /\left(\omega^{2}-\omega_{A, T O}^{2}\right)$, as for alkali halide or polar semiconductor materials, where $\omega_{A, L O}$ and $\omega_{A, T O}$ are the longitudinal and transverse optical frequencies, respectively. As model parameters, we take $\varepsilon_{B}=2.1$, as the value of $\mathrm{SiO}_{2} ; \varepsilon_{A, \infty}=2.34, \varepsilon_{A, 0}=5.9, \omega_{A, T O}=$ $32.01 \mathrm{THz}$ and $\omega_{B, L O}=50.74 \mathrm{THz}$, which correspond to the values of $\mathrm{NaCl}$; finally, $\varepsilon_{C}=1$, representing the dielectric constant of vacuum.

Figure 1 shows the calculated dispersion relation of coupled optical interface modes for a 9th order TM dielectric superlattice, where $d=2 d_{S}, d_{L}=1.5 d_{S}$, and $d_{S}$ is fixed. The spectrum is divided into two branches, namely $\omega_{+}$and $\omega_{-}$, which are separated by a gap just as in the cases of semi-infinite periodic ${ }^{10)}$ and quasiperiodic Fibonacci ${ }^{17)}$ superlattices. For smaller $k d_{S}$, the spectrum forms two bands, while for larger $k d_{S}$, the modes are highly degenerate; and $\omega_{+}$and $\omega_{-}$approach a same 


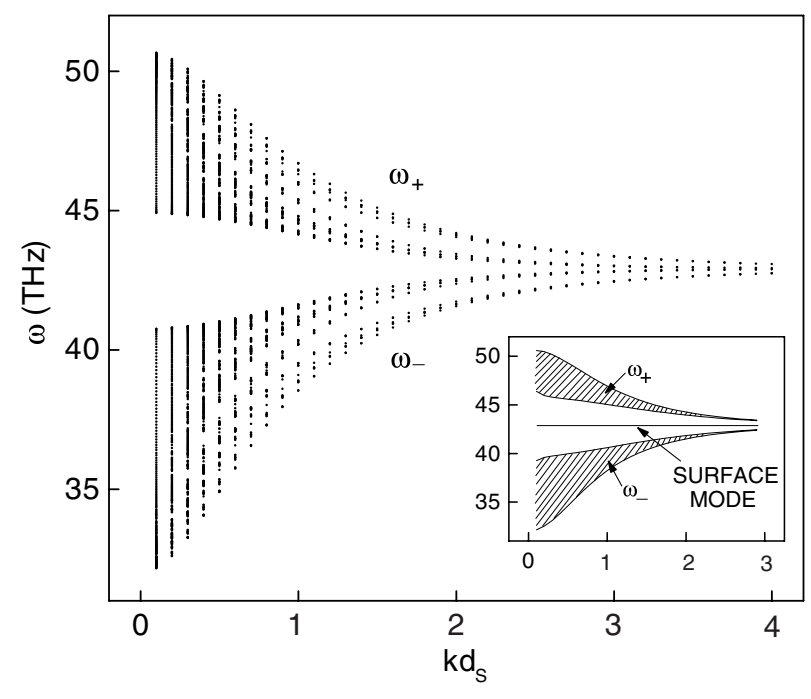

Fig. 1. Dispersion relation of the coupled optical interface modes for a 9 th order TM superlattice with $d=2 d_{S}$ and $d_{L}=1.5 d_{S}$. Inset, dispersion relation of the coupled optical interface modes for a semiinfinite periodic superlattice with $d=2 d_{L}=2 d_{S}$.

limiting frequency $\omega_{0}=42.912506823674 \mathrm{THz}$, which satisfies the equation $\varepsilon_{A}\left(\omega_{0}\right)=-\varepsilon_{B}$.

Specifically, if we choose $d_{L}=d_{S}=d_{A}$ and $d=d_{B}$, the aperiodic TM superlattice turns into a periodic one. It is well known that for infinite periodic dielectric superlattices, when $d_{A}$ and $d_{B}$ are small enough, the eigenfrequencies exist in the region where $\varepsilon_{A} / \varepsilon_{B}$ is negative. In fact, $\omega_{+}$and $\omega_{-}$continuosly cover all the region between $\omega_{A, L O}$ and $\omega_{A, T O}$ and the frequency $\omega_{0}$ is just the demarcation point of two branches. ${ }^{10)}$ But for the semi-infinite periodic superlattice, a gap emerges between $\omega_{+}$and $\omega_{-}$and a surface mode appears in the gap, ${ }^{10)}$ as shown in the inset. However, we can see from Fig. 1 now that for a TM superlattice, besides the largest gap between $\omega_{+}$and $\omega_{-}$, there are many other small gaps to appear amid $\omega_{+}$or $\omega_{-}$branches.

Compared with the spectra of a Fibonacci dielectric superlattice, ${ }^{17}$ ) although there is a similarity for both are singular continuous, which means that the number of states whose eigen-frequencies are less than a specified frequency $\omega$ is continuously increasing but non-differentiable at any $\omega$, just like the Cantor set, the spectra gaps here have completely different branching rules. Moreover, when the generation number increases, the gap number of a TM dielectric superlattice rises faster than that of a Fibonacci superlattice does. These differences reflect the underlying characteristics of relevant sequences. ${ }^{7)}$ For clearness, we would pay much attention to $k d_{S}=0.5$ in Fig. 1, then a plot for frequency vs number of the modes is shown in Fig. 2. It can be seen from the spectrum that the allowed frequencies form two branches, which are all singular continuous. Our detailed investigation finds that every branch is divided into six subbands. The first (sixth) and third (fourth) subbands are formed by $S S(L L)$ clusters and other subbands are formed by isolated $S$ or $L$ blocks. Each subbands has approximately the same number of modes and is to split further. Just as shown in the insets of Fig. 2, the second

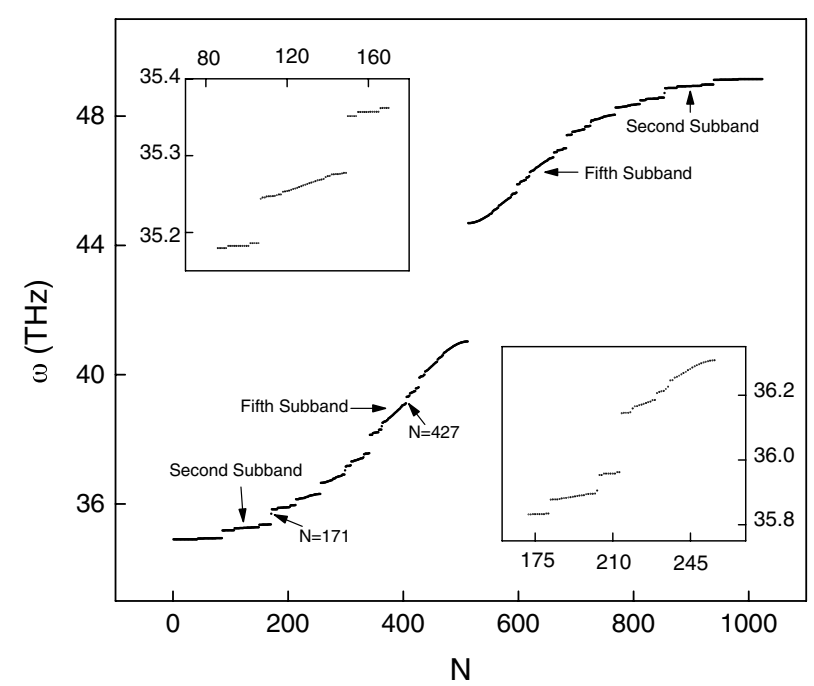

Fig. 2. Eigenfrequency versus number of modes for a 9th order TM superlattice with $k d_{S}=0.5, k d_{L}=0.75$ and $k d=1.0$. The modes $N=171$ (in the gap) and $N=427$ (on the edge of a subband) are indicated. Inset at top-left, enlargement of the second subband of $\omega_{-}$ branch. Inset at foot-right, enlargement of the third subband of $\omega$ branch.

and the fifth subbands, respectively, are trifurcate and the others are bifurcate. All these are consistent with the general rule of branching for electronic energy spectra of TM chains, ${ }^{18,20)}$ which is obtained in the on-site framework under the periodic-boundary conditions. Obviously, the branching rule of the spectra shown here is different from that of the Fibonacci superlattice, ${ }^{17)}$ which is only trifurcate.

Except the modes in subbands, there are still a few frequencies locating in the gaps. The mode $N=171$ in Fig. 2 is such an example. Noting that in the Fibonacci superlattice they also exist, we consider that they only stem from the boundary conditions taken here and do not reflect the intrinsic character of TM sequence itself. So it is reasonable to expect that these modes will disappear under the periodic approximation. Our research also shows that these frequencies lead to electrostatic potential distributions with oscillatory decayed amplitudes near the surfaces.

The singular continuity of the spectrum can be displayed effectively by calculating the IDOS. Although the density of states is not well-defined in the singular continuous spectrum, the IDOS, namely the total number of states with eigen-frequencies less than $\omega$, is always well-defined just as in the absolutely continuous spectrum. Because the negative-eigenvalue method seems inapplicable here, we have obtained IDOS in a poorman's way. Fig. 3 gives a result for the 9th order TM superlattice. However, for $j=7,8$ and $j=10$, the basic features are unchanged. To understand the result and label the gaps in Fig. 3, we refer the previous work of Luck, ${ }^{7)}$ Tamura and Nori. ${ }^{19)}$ In ref. 7 , Luck performed a perturbative analysis for the IDOS of the electronic energy spectrum of general diterministic aperiodic systems, and found that the gaps can be labeled by the singularities of the Fourier transform of the sequence 


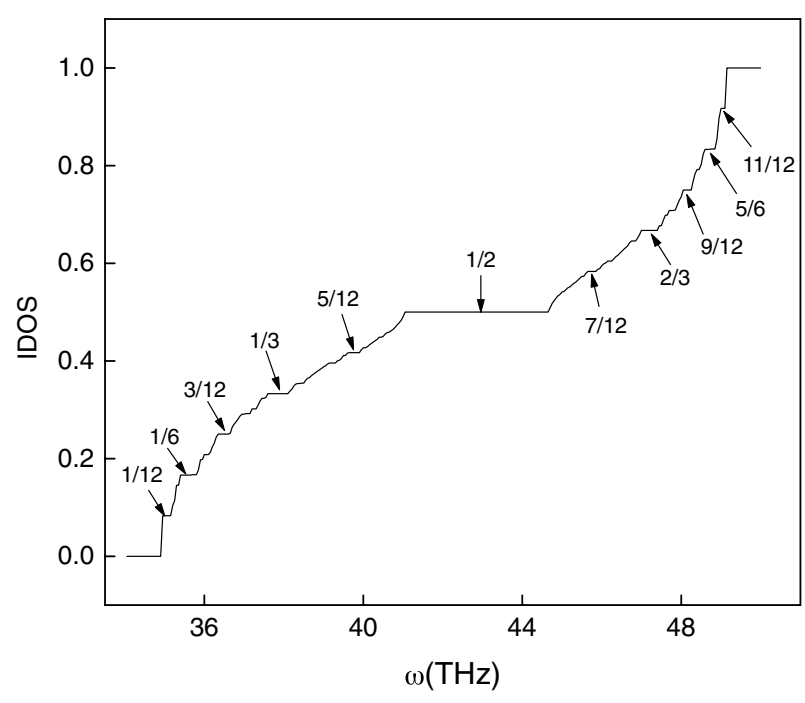

Fig. 3. The IDOS for a 9th order TM superlattice with the parameters chosen in Fig. 2.

with on-site potentials. Simultaneously, in ref. 19, Tamura and Nori derived the structure factor of TM superlattices and used it to describe the interference effects of phonons reflected at the interface of consititute layers. They established the label theorem of gaps in the acoustic phonon frequency spectra and predicted the locations of the transmission dips by the property of the structure factor. All their results can be summarized as that, in the energy or frequency spectra of a TM sequence, there is a gap at the value of the IDOS equal to $(2 k+1) /\left(3 \times 2^{p}\right)$ with non-negative integer $k$ and $p$. The similar conclusion was also obtained by Axel and Peyriere at the same time. $\left.{ }^{9}\right)$ Following the cited results, we can label the most visible gaps, as shown in Fig. 3, and the indices have one to one correspondences to the Luck, Tamura and Nori's results, although which are obtained for electrons and acoustic phonons by taking the periodic-boundary conditions, and our result of optical interface modes is achieved under the freeboundary conditions. It suggests that the free boundaries considered here have little effect on the basic features of the frequency spectrum. This is also confirmed by the calculations of electrostatic potential profiles. The reason may be that, for $j=9$, the TM superlattice has $2^{j+1}(=1024)$ layers, so it is thick enough to exhibit the entitative feature of TM sequences.

For comparison, we have also calculated the corresponding IDOS for periodic and quasiperiodic Fibonacci superlattices, and the spectrum of the later is no doubt singular continuous, which is similar, except that the gap values are different, to the TM structure, but both have radical difference with the periodic system. In periodic case, gaps will disappear when the superlattices go to infinity, ${ }^{10)}$ but in deterministic aperiodic superlattices these gaps will persist even in infinite systems. ${ }^{7,8,19)} \mathrm{We}$ consider that this fact supports the prediction of Luck: energy spectra associated with deterministic aperiodic sequences seem to be always a Cantor set of zero Lebesque measure. Furthermore, it should be noted that the above gap label theorem for TM sequences have

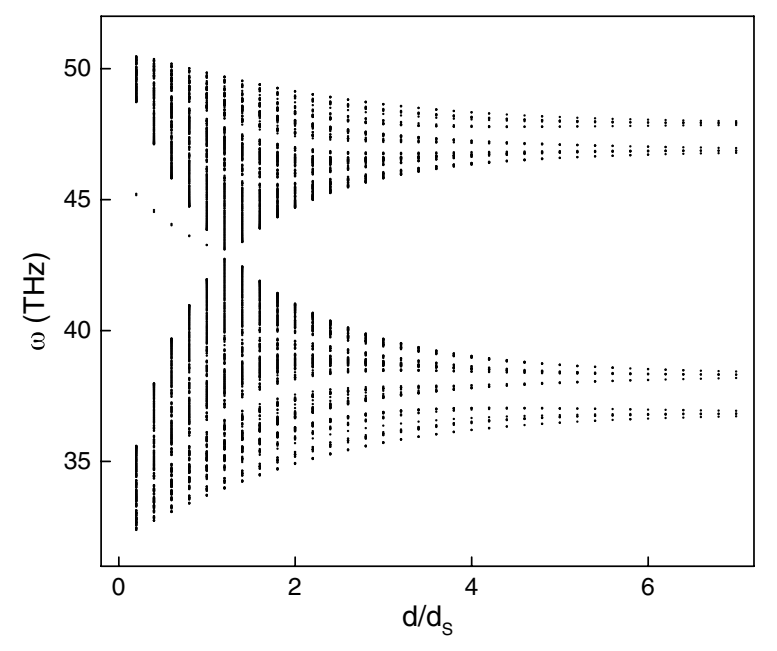

Fig. 4. The variation of eigenfrequency distribution with the thickness ratio $d / d_{S}$ for $k d_{S}=0.5$ and $k d_{L}=0.75$.

already been proved thoroughly by the high-resolution X-ray diffraction spectra. ${ }^{25)}$

The relative thicknesses of $d, d_{L}$ and $d_{S}$ have important effects on the frequency spectra. One example is shown in Fig. 4, where $k, d_{L}$ and $d_{S}$ are fixed $\left(k d_{S}=\right.$ $\left.0.5, k d_{L}=0.75\right)$ but $d$ is changeable. For $d / d_{S} \ll 1$, the eigenfrequencies approach $\omega_{A, L O}$ and $\omega_{A, T O}$, while for $d / d_{S} \gg 1$, there exit four limiting frequencies $\omega=36.836$ $630462942 \mathrm{THz}, 38.300481939860 \mathrm{THz}, 46.876310$ $152912 \mathrm{THz}$, and $47.908930619391 \mathrm{THz}$. These four limiting frequencies represent the isolated modes when $d$ approaches infinity, so they actually correspond to the case where an isolated $A$ dielectric layer with $d_{L}$ or $d_{S}$ thickness is embeded in the infinite $B$ media. Analysis shows that these frequencies are exactly the solutions of the following four equations

$$
\begin{array}{ll}
\varepsilon_{A}=-\varepsilon_{B} \tanh \left(k d_{S} / 2\right), & \varepsilon_{A}=-\varepsilon_{B} \operatorname{coth}\left(k d_{S} / 2\right), \\
\varepsilon_{A}=-\varepsilon_{B} \tanh \left(k d_{L} / 2\right), & \varepsilon_{A}=-\varepsilon_{B} \operatorname{coth}\left(k d_{L} / 2\right) .
\end{array}
$$

Figure 5 gives an another example, where $k, d$ and $d_{S}$ are fixed, but $d_{L}$ varies. Notice that, for $d_{L} / d_{S} \rightarrow 1$, two continuous bands are there, which root from the fact that the structure becomes periodic. However, the deterministic aperiodicity is more prominent for small or large values of $d_{L} / d_{S}$. Interestingly, we can find that the odd or even of the generation number $j$ seriously affects the frequency spectra, and the reason is that when $j$ is even (odd), the TM structure is symmetric (antisymmetric). Particularly for large $d_{L} / d_{S}$, if $j$ is even, the spectra become thirteen highly degenerate branches, which correspond to a kind of isolated modes. These limiting frequencies satisfy a set of equations.

$$
\begin{aligned}
& \varepsilon_{A}=-\varepsilon_{B} \frac{\varepsilon_{B} \tanh k d+\varepsilon_{C}}{\varepsilon_{B}+\varepsilon_{C} \tanh k d}, \\
& \varepsilon_{A}=-\varepsilon_{B} \tanh (k d / 2), \quad \varepsilon_{A}=-\varepsilon_{B} \operatorname{coth}(k d / 2), \\
& \alpha^{2} \mathrm{e}^{k d_{S}}-\beta^{2} \mathrm{e}^{-k d_{S}}=0, \\
& \alpha^{3} \mathrm{e}^{2 k d_{S}}-2 \alpha \beta^{2}-\beta^{2} \gamma \mathrm{e}^{-2 k d_{S}}=0,
\end{aligned}
$$

where $\alpha, \beta$ and $\gamma$ are defined as in eqs. (5) by substituting $d_{l}$ with $d$. On the other hand, if $j$ is odd, 


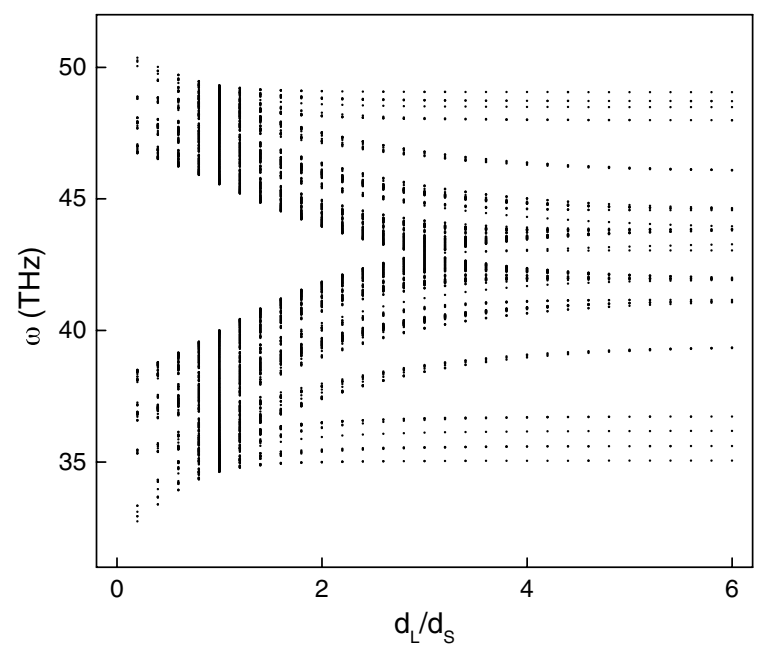

Fig. 5. The variation of eigenfrequency distribution with the thickness ratio $d_{L} / d_{S}$ for $k d_{S}=0.5$ and $k d=1.0$.

there exist sixteen limiting frequencies, just as in Fig. 5, which also satisfy eqs. (15), but another equation

$$
\begin{aligned}
\left(\varepsilon_{B}-\varepsilon_{C}\right)\left(\varepsilon_{B}+\varepsilon_{A}\right) \alpha_{S} \mathrm{e}^{2 k d} & -\left(\varepsilon_{B}+\varepsilon_{C}\right)\left(\varepsilon_{B}-\varepsilon_{A}\right) \gamma_{S} \mathrm{e}^{-2 k d} \\
& +2 \beta_{S}\left(\varepsilon_{B}^{2}+\varepsilon_{A} \varepsilon_{C}\right)=0
\end{aligned}
$$

should be added.

It is instructive to point out that in Figs. 4 and 5, if one of the thicknesses $d, d_{L}$ and $d_{S}$ is larger than the rest two with several times, the overlap of macroscopic electric field induced by optical vibrations in neighboring blocks becomes very weak, so the limiting isolated modes will emerge. These isolated interface modes for artificial TM superlattices may be detected by experiments such as the Raman scattering ${ }^{11)}$ or electron energy-loss spectra $^{26)}$ measurement.

\section{§4. Electrostatic Potential Profiles}

The characteristics of the frequency spectra arising from the deterministic aperiodic structure should be reflected in the distributions of electrostatic potentials, which are related to the long-wavelength optical vibrations. Actually, these potential distributions are important to theoretical investigation of Raman scattering. ${ }^{4}$ For a simplified description here, we are only concerned with the averaged potential over each layer, then

$$
\phi_{l}^{B}=\frac{\sinh (k d / 2)}{k d / 2}\left(p_{l}+q_{l}\right)
$$

for the $B$ layers, and

$$
\begin{aligned}
\phi_{l}^{A}= & \frac{1}{k d_{l}}\left\{\left[\sinh k d_{l}+\frac{\varepsilon_{B}}{\varepsilon_{A}}\left(\cosh k d_{l}-1\right)\right] \mathrm{e}^{k d / 2} p_{l}\right. \\
& \left.+\left[\sinh k d_{l}-\frac{\varepsilon_{B}}{\varepsilon_{A}}\left(\cosh k d_{l}-1\right)\right] \mathrm{e}^{-k d / 2} q_{l}\right\}
\end{aligned}
$$

for the $A$ layers.

For the parameters chosen in Fig. 2, we have examined all the potential profiles of 1024 eigenfrequencies and find that almost all states are extended and lattice-like. ${ }^{20,21)}$ It is completely different with the
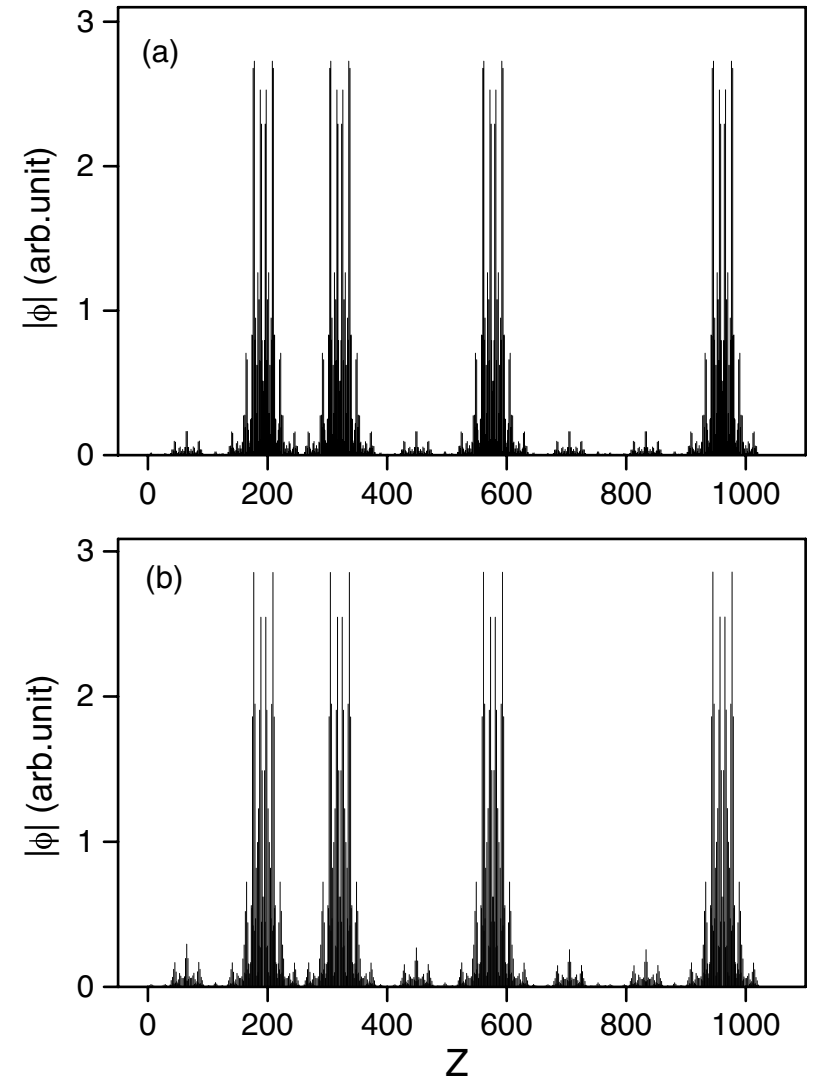

Fig. 6. The electrostatic potential profiles for two dual lattice-like states, (a) $\quad \omega=34.926899831760 \mathrm{THz} ; \quad$ (b) $\quad \omega=$ 49.125801668610 $\mathrm{THz}$.

results obtained from quasiperiodic Fibonacci superlattice, ${ }^{17)}$ where the potential profiles are critical and self-similar. Two lattice-like states, which correspond to $N=48$ and 977 modes in Fig. 2, are given in Figs. 6(a) and $6(\mathrm{~b})$. From these two figures we can at least understand two unambiguous facts. The first is that the spatial amplitude distribution in each potential profile forms a TM sequence. It has been known that the similar wavefunctions of electrons in TM sequence were found earlier by numerical calculations ${ }^{20}$ ) and then discussed by analytical method. ${ }^{21)}$ In fact, under Bornvon Karman boundary conditions, we can rigorously prove that all eigenstates of TM sequences are extended, which means the amplitudes are nonzero at infinity, and lattice-like, which means the array of the amplitudes forms a TM arrangement. So the optical vibrations in TM dielectric superlattices form deterministic but nonperiodic extended states. This conclusion is applicable to other elementary excitations in TM sequences, such as electrons and acoustic phonons. By the way, the eigenfrequencies obtained numerically from the 9th TM superlattice can be used to get the potential profiles of TM superlattices for $j=10,11, \ldots$, with their potential distributions keeping TM behavior as shown in Fig. 6. This illustrates the fact that the boundary conditions are unimportant and the frequency spectra of lower order will be included in those of higher order of TM superlattices.

Secondly we can notice that $N=977$ mode is the 48 th 
mode counted from the upper part of the $\omega_{+}$band. The similarity of these two states denotes duality of the $\omega_{-}$ and $\omega_{+}$bands, which is also found for optical phonons in Fibonacci superlattices. ${ }^{17)}$ Actually, we found that almost all the states for these two bands are exactly one-to-one correspondent in overall characteristics, although there may be some small differences in detail. The duality indicates a pronounced difference of optical phonons from eletrons and acoustic phonons. Our further work shows that this property of the frequency spectra and the potential profiles comes from the frequency dependence of the dielectric function $\varepsilon_{A}$ through the symmetry of the transfer matrix defined in eqs. (4) and (5). Based on this consideration, we can expect that if the dielectric function $\varepsilon_{B}$ is also frequency dependent, the frequency spectra will be quartfurcate, and the relevant four frequencies from four bands will give the similar electrostatic potential profiles. This quartet in the characteristics of frequency spectra and potential distributions have really been confirmed by numerical calculations.

Returning to the previous discussion, Fig. 7 shows the potential profile of the mode $N=1$ (its dual mode is $N=1024$ ) in Fig. 2. It has its analogue for the wavefunction of an electron in TM chain, ${ }^{18)}$ and seems stranger than the case in Fig. 6. The potential profile in this figure looks like that of composed of several smooth envelope curves, which are quite similar to the case of an electron bounded in a quantum well. So, for the first glance, it may bring some suspicions to say this kind of eigenstate is also lattice-like. ${ }^{18)}$ However, our detailed analysis shows that the distribution of the electrostatic potential is an overlap of several components, each of which obeys the TM sequence strictly. Moreover, if we enlarge the scope of the potential distribution with the same frequency, the TM arrangement in a large size will appear clearly. These do let us believe that the potential profile in Fig. 7 is lattice-like in nature.

Among the 1024 modes, there are still a few giving surface states as shown in Fig. 8. These two states are localized at the surfaces of the superlattices. Noted that in an infinite TM harmonic chain there are only extended

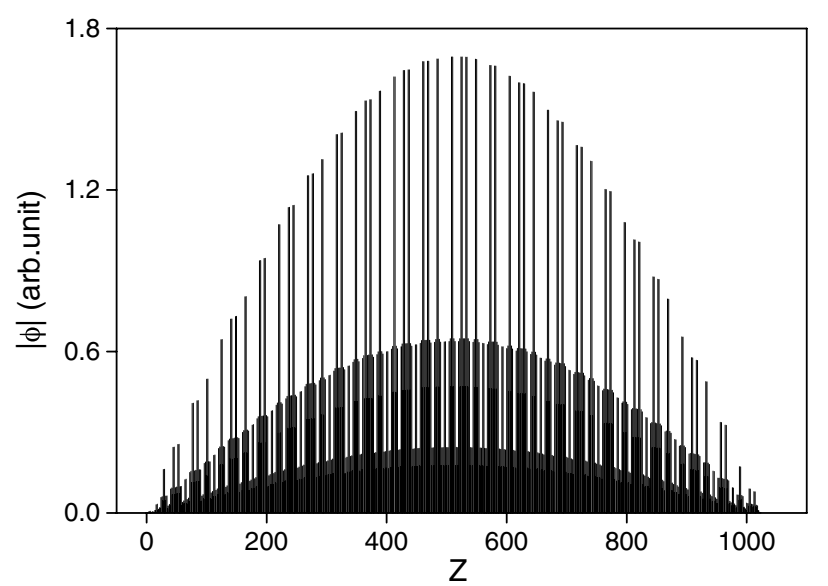

Fig. 7. The electrostatic potential profile with $\omega=$ 34.900339707022 $\mathrm{THz}$ forming envelope curves.
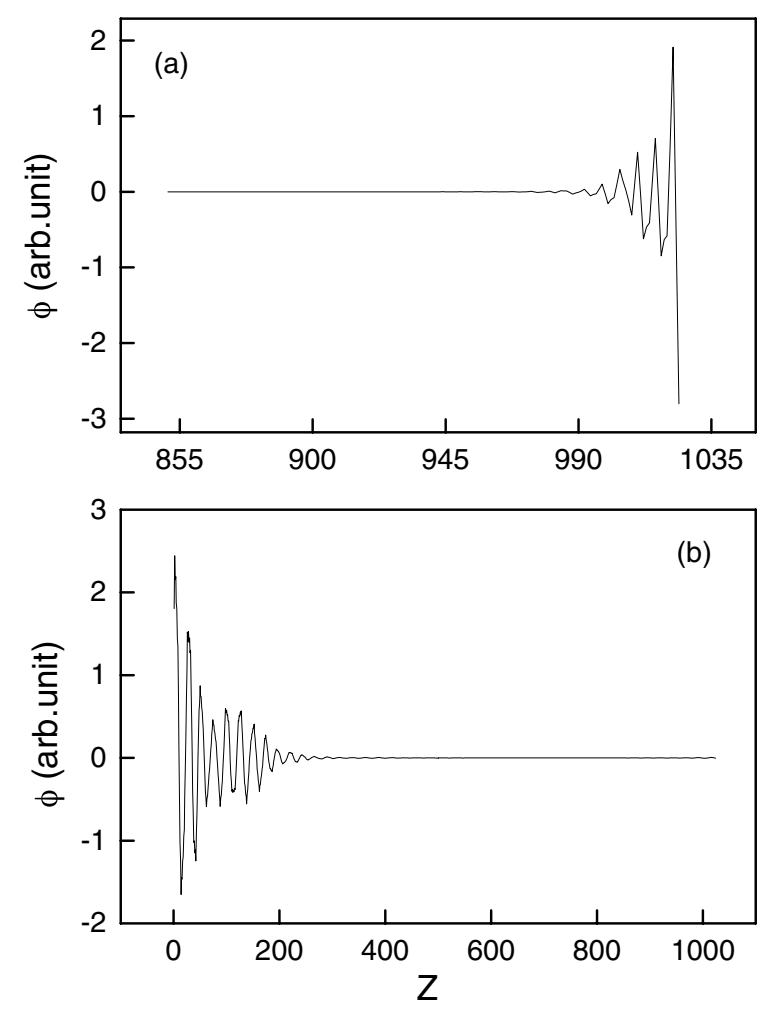

Fig. 8. The electrostatic potential profiles for two localized states, (a) $\omega=35.695204831121 \mathrm{THz}$; (b) $\omega=39.605606631415 \mathrm{THz}$.

states, ${ }^{9)}$ the apparent difference indicates that the finiteness of the structure examined here has some additional effects. These surface modes, which originate from the boundary conditions considered here, usually locate at the edges of the subbands or in the gaps in Fig. 2. The frequencies for Fig. 8 are corresponding to the $N=171$ and 427 modes, and the former is in the gap between the second and the third subbands of the $\omega_{-}$ branch while the later is at the edge of a subband exactly. Furthermore, we can find that the modes in the gap are much localized than the modes at the band edge, as clearly shown in the Figs. 8(a) and 8(b).

\section{§5. Summary}

We have studied the coupled optical interface modes in a finite TM dielectric superlattice. By taking into account of the long wave approximation, a theoretical scheme has been developed based on the transfer matrix method. The eigen-equation for frequency spectra was given and the electrostatic potentials can be obtained recursively.

The numerical results have shown, as expected, that the eigenfrequencies all locate between the longitudinal and transverse frequencies of the bulk material, and appear as a singular continuous set, which resembles the spetra of Fibonacci superlattices, but completely differs from the results of periodic structures, whose spectra is absolutely continuous. Although the frequency spectra of TM dielectric superlattices look like those of Fibonacci ones, there are two fundamental differences, including gap lables and branching rules, which just reflect the distinct structure features of two sequences. Our results 
also have shown that the electrostatic potential profiles of TM superlattices are almost all extended and latticelike, which is consistent with the results from periodic boundary conditions. Noting that the eigenmodes of a periodic structure are Blochwaves and of a Fibonacci superlattice are critical states, accordingly, we may say that Thue-Morse structure is intermediate between periodic structure and Fibonacci structure. However, due to the singular continuity of the Fourier spectra of TM sequences, ${ }^{7,8)}$ the judgement is still not so confident. This dilemma gives the reason why investigation of the physical properties in TM structures has been attractive so far. ${ }^{18-23)}$ Furthermore, except a few surface modes, the boundary conditions have little affection on the properties of elementary excitations, so the experiments performed on the optical phonons in finite TM superlattices are expected to expose the intrinsic nature of infinite TM sequence.

Compared with eletrons and acoustic phonons, it is clear that the optical phonons have the common features of the elementary excitations in TM structures, which mainly refer that firstly the frequency (energy) spectra are singular continuous sets having same branching rules and gap lables, and secondly the eigenstates are extended and lattice-like. This analogue is natural in the sense that all are involved in wave propagations in TM sequences. On the other hand, the coupled optical interface modes have their own unique characteristic, i.e., the duality or even quartet of the frequency spectra and the potential profiles. Moreover, it should be emphasized that dielectric superlattices can be manufactured nicely with nowaday technology, so experimental observation may be fulfilled much conveniently by Raman light scattering $^{11)}$ or X-ray diffraction measurement ${ }^{25)}$ or electron energy-loss spectra, ${ }^{26)}$ and then the elementary excitation properties in aperiodic TM structures will be illustrated.

\section{Acknowledgments}

This work was supported by the National Natural Science Foundation of China, the State Key Program for Basic Research from the Ministry of Science and
Technology of China, and the key research project in the "Climbing Program" by the National Science and Technology Commission of China.

The authors are gratefull to the Physical Society of Japan for financial support in publication.

1) R. Merlin, K. Bajema, R. Clarke, F.-Y. Juang and P. K. Bhattacharya: Phys. Rev. Lett. 55 (1985) 1768.

2) A. Hu, C. Tien, X. Li, Y. Wang and D. Feng: Phys. Lett. A 119 (1986) 313.

3) M. Kohmoto, B. Sutherland and C. Tang: Phys. Rev. B 35 (1987) 1020.

4) P. Hawrylak and J. J. Quinn: Phys. Rev. Lett. 57 (1986) 380.

5) J. W. Feng, G. J. Jin, A. Hu, S. S. Kang, S. S. Jiang and D. Feng: Phys. Rev. B 52 (1995) 15312.

6) G. Gumbs and M. K. Ali: Phys. Rev. Lett. 60 (1988) 1081.

7) J. M. Luck: Phys. Rev. B 39 (1989) 5834.

8) Z. Cheng, R. Savit and R. Merlin: Phys. Rev. B 37 (1988) 4375.

9) F. Axel and J. Peyriere: J. Stat. Phys. 57 (1989) 1013.

10) R. E. Camley and D. Mills: Phys. Rev. B 29 (1984) 1695.

11) A. K. Sood, J. Menendez, M. Cardona and K. Ploog: Phys. Rev. Lett. 54 (1985) 2115

12) Kun Huang and Bangfen Zhu: Phys. Rev. B 38 (1988) 13377.

13) S. Tamura and J. P. Wolfe: Phys. Rev. B 38 (1988) 5610.

14) M. Kohmoto, B. Sutherland and K. Iguchi: Phys. Rev. Lett. 58 (1987) 2436.

15) W. Gellermann, M. Kohmoto, B. Sutherland and P. C. Taylor: Phys. Rev. Lett. 72 (1994) 633.

16) T. Hattori, N. Tsurumachi, S. Kawato and H. Nakatsuka: Phys. Rev. B 50 (1994) 4220.

17) G. J. Jin, S. S. Kang, Z. D. Wang, A. Hu and S. S. Jiang: Phys. Rev. B 54 (1996) 11883.

18) R. Riklund, M. Severin and Youyan Liu: Int. J. Mod. Phys. B 1 (1987) 121.

19) S. Tamura and F. Nori: Phys. Rev. B 40 (1989) 9790.

20) C. S. Ryu, G. Y. Oh and M. H. Lee: Phys. Rev. B 46 (1992) 5162; ibid. 48 (1993) 132.

21) A. Chakrabarti, S. N. Karmakar and R. K. Moitra: Phys. Rev. Lett. 74 (1995) 1403.

$22)$ M. S. Vasconcelos and E. L. Albuquerque: Phys. Rev. B 57 (1998) 2826; ibid. 59 (1999) 11128.

23) A. Ghosh and S. N. Karmakar: Phys. Rev. B 58 (1998) 2586; ibid. $61(2000) 1051$.

24) M. Kolar and M. K. Ali: Phys. Rev. B 42 (1990) 1062.

25) F. Axel and H. Terauchi: Phys. Rev. Lett. 66 (1991) 2223.

26) Ph. Lambin, J. P. Vigneron, A. A. Lucas, P. A. Thiry, M. Liehr, J. J. Pireaux, R. Caudano and T. J. Kuech: Phys. Rev. Lett. 56 (1986) 1842. 